\title{
Research on Robust Task Space Control Uncertainty Based on Legendre Polynomial
}

\author{
Xinwen $\mathrm{Bi}^{1,}$, Zeliang Zhang ${ }^{1, \mathrm{~b}^{*}}$ and Ming $\mathrm{Ma}^{1, \mathrm{c}}$ \\ ${ }^{1}$ Institute of Information Technology and Media, Beihua University, Jilin City, China \\ a52600613@qq.com, ${ }^{b} 11701552 @ q q . c o m,{ }^{c} 931406363 @ q q . c o m$ \\ *The corresponding author
}

Keywords: Robust control; Legendre polynomials; Sure border; Voltage control strategy

\begin{abstract}
Usually, the uncertainty bound is needed to design the control law in conventional robust control approaches. However, the proposed bound may increase the amplitude of the control signal and damage the system. To solve this problem, a robust control law is proposed in this paper. The uncertainty bound of the proposed control law is calculated by Legendre polynomials. Compared to conventional robust controllers, the proposed controller is simpler, less computational and requires less feedback. By a SCARA robot manipulator control law proposed simulation, the simulation results verify the effectiveness of the proposed control approach.
\end{abstract}

\section{Introduction}

In the past decades, the adaptive control and robust control of robot manipulators have been extensively studied in task space [1] and joint space [2]. Robust and adaptive control are considered important because they can overcome the uncertainty between the nominal model and the actual model due to mismatches. External disturbances, parametric uncertainties and unmodeled dynamic characteristics are the main sources of uncertainty in control engineering, and also seriously affect the performance of the controller.

In the early studies of robust control methods [3], controller designs are often based on nominal models. A robust control term is then added to the control law to compensate for the uncertainty, which needs to be determined by the Lyapunov stability analysis. In these methods, uncertain boundaries need to be used to determine the stability of the system and to design the control law. Normally this boundary is the upper limit of the system state and external disturbance. Therefore, all required feedback should be available, and the upper limit of parameter uncertainty and external interference should be known in advance. In addition, the linear parameterization of the manipulator kinematics equation is necessary in most robust and adaptive control methods [4]. The controller motion equation should be modeled completely to determine the regression matrix.

Most of the research in the field of robot control is based on the torque control strategy (TCS). But often TCS ignores the dynamic performance of the drive. To solve this problem, a simple and convenient voltage control strategy (voltage-based controller, VCS) was proposed. Voltage-based manipulator controller stability analysis has been studied [5]. Based on the VCS, scholars have proposed different robust control methods [6,7].

Recently, a number of adaptive control methods for regressions have been proposed [8], and the uncertainty has been estimated using the Fourier series. Based on the Lyapunov stability, some adaptive rules are deduced to adjust the Fourier series coefficients. According to [9], some other orthogonal functions, such as Legendre and Chebyshev polynomials, can be approximated to continuous time functions at arbitrary precision. In this paper, we use this idea to estimate the uncertain boundary of the Robot Task Space Control for the electrodynamic robot.

Based on the above analysis, this paper presents a more simple method compared with the literature $[10,11]$. In this paper, the lumped uncertainty for each joint is estimated. Another 
advantage of the proposed model is that it eliminates the need to know in advance the conditions for external disturbances and the upper limit of the parameter uncertainty.

\section{A Traditional Robust Control Method Using VCS}

According to [12], a robot system including a manipulator and a permanent magnet DC motor can be described by the following nonlinear state space equation.

$$
\dot{\mathrm{Z}}=F(\mathrm{Z})+\left[\begin{array}{lll}
0 & 0 & L^{-1}
\end{array}\right]^{T} \mathrm{v}
$$

The above equation $Z=\left[\begin{array}{lll}q & \dot{q} & I_{a}\end{array}\right]^{T}$ is the state vector, $q \in R^{n}$ is the joint position vector, $\dot{q} \in R^{n}$ is the joint space velocity vector, $I_{a} \in R^{n}$ is the motor current vector, $\mathrm{L}$ is $\mathrm{n} \times \mathrm{n}$ diagonal matrix, it is the armature inductance coefficient, $\mathrm{n}$ is the number of joints. The nonlinear vector function $\mathrm{F}(\mathrm{Z})$ is as follows:

$$
F(Z)=\left[\begin{array}{l}
Z_{2} \\
\left(J_{m} r^{-1}+r D\left(Z_{1}\right)\right)^{-1}\left(-\left(B_{m} r^{-1}+r C\left(Z_{1}, Z_{2}\right)\right) Z_{2}-\right. \\
r G\left(Z_{1}\right)+K_{b} Z_{3} \\
-L^{-1}\left(K_{b} r^{-1} Z_{2}+R Z_{3}\right)
\end{array}\right]
$$

$D\left(Z_{1}\right)$ is the inertial $\mathrm{n} \times \mathrm{n}$ matrix of the manipulator, $C\left(Z_{1}, Z_{2}\right) Z_{2} \in R^{n}$ is the centrifugal force and Coriolis force vector, $G\left(Z_{1}\right) \in R^{n}$ is the gravity moment vector, $J_{m}, B_{m}$, and $\mathrm{r}$ are the diagonal matrix of the motor inertia, damping and deceleration gear coefficients, respectively. Is the motor voltage vector, and $\mathrm{n} \times \mathrm{n}$ diagonal matrix representing the armature resistance and counter electromotive force constant coefficients, respectively. In this paper, it is assumed that the nonlinear vector function is unknown.

The position and velocity in the task space are denoted by $X \in R^{3}$ and $\dot{X} \in R^{3}$ respectively. The Jacobian matrix associates $J(q) \in R^{3 \times n}$ the joint space velocity vector $\dot{q}$ with the task space velocity vector $\dot{X}$ as follows:

$$
\dot{X}=J(q) \dot{q}
$$

The matrix form of the electrical equations of a permanent magnet DC motor is as follows:

$$
R I_{a}+L \dot{I}_{a}+K_{b} r^{-1} \dot{q}+\varphi=v
$$

$\varphi \in R^{n}$ is the external disturbance vector, which can be obtained by [9]. This paper assumes that the manipulator runs in a range where $J^{-1}(q)$ is nonsingular. (4) can be written as: $R I_{a}+L \dot{I}_{a}+K_{b} r^{-1} J^{-1}(q) \dot{X}+\varphi=v$

Suppose $\hat{K}_{b}, \hat{r}$ and $\hat{J}(q)$ represent the nominal values of $K_{b}, r$ and $J(q)$, respectively. By increasing and decreasing $\hat{K}_{b} \hat{r}^{-1} \hat{J}^{-1}(q) \dot{X}$, Equation (5) can be given by:

$$
\hat{K}_{b} \hat{r}^{-1} \hat{J}^{-1}(q) \dot{X}+F(t)=v
$$

Here $F(t)$ is the lumped uncertainty. From (5) and (6) can be derived: 


$$
\begin{aligned}
F(t)= & R I_{a}+L \dot{I}_{a}+K_{b} r^{-1} J^{-1}(q) \dot{X} \\
& +\varphi-\hat{K}_{b} \hat{r}^{-1} \hat{J}^{-1}(q) \dot{X}
\end{aligned}
$$

As stated earlier, undetermined boundaries are required in most robust control methods.

Therefore, suppose that the bounded uncertainty function $F(t)$ in (7) is $\|F(t)\| \leq \eta(t)$, where the scalar function $\eta(t)$ is given by:

$$
\begin{aligned}
& \eta(t)=\|R\|\left\|I_{a}\right\|+\|L\|\left\|\dot{I}_{a}\right\|+ \\
& \left\|K_{\mathrm{b}} r^{-1} J^{-1}-\hat{K}_{b} \hat{r}^{-1} \hat{J}^{-1}\right\|\|\dot{X}\|+\|\varphi\|
\end{aligned}
$$

A robust control law is proposed for the system (6) as follows:

$$
\hat{K}_{b} \hat{r}^{-1} \hat{J}^{-1}(q)\left(\dot{X}_{d}+K_{p} \tilde{X}\right)+u_{r}=v
$$

Where $\tilde{X}=X_{d}-X$ is the mission space tracking error, $X_{d}$ is the desired trajectory, $K_{p}$ is proportional to the gain of the symmetric matrix, $u_{r}$ is a robust control, which will be determined later.

\section{Control Rules Put Forward.}

The robust term $u_{r}$ [9] requires that the boundary $\eta$ is not determined. According to Eq. (8), $\eta$ is a function of the motor current $I_{a}$, its derivative $\dot{I}_{a}$ and the mission space velocity $\dot{X}$. These signals should be measured and fed back to the controller. In most real-time applications, the motor current and its derivatives of time are overwhelmed by noise. So their use in the control law may reduce the performance of the controller. In addition, the maximum values of $\|R\|,\|L\|$, $\left\|K_{b} r^{-1} J^{-1}-\hat{K}_{b} \hat{r}^{-1} \hat{J}^{-1}\right\|$, and $\|\varphi\|$ are required. To solve this problem, the uncertain boundary $\eta$ is estimated by LPs.

Closed-loop system, can be written as:

$$
\hat{K}_{r} \hat{J}(q)^{-1}\left(\dot{\tilde{X}}+K_{p} \tilde{X}\right)=\left(F-u_{r}\right)
$$

According to the orthogonal function theorem [9], the uncertain boundary $\eta(t)$ in Eq. (8) can be estimated by Legendre polynomial:

$$
\hat{\eta}(t)=\hat{P}^{T} \xi(t)
$$

Where $\hat{P}=\left[\begin{array}{llll}\hat{a}_{0} & \hat{a}_{1} & \ldots \hat{a}_{m}\end{array}\right]^{T}$ is the vector estimation, $P^{*}=\left[\begin{array}{lll}a_{0} & a_{1} & \ldots a_{m}\end{array}\right]^{T}$ and $(\mathrm{i}=0,1, \ldots$, $\mathrm{m})$ are the optimal values of the Legendre coefficients, The estimation error $|\eta-\hat{\eta}|$ is reduced. In order to get the adaptive rule of $\hat{P}$, consider the following positive definite function.

$$
L=\frac{1}{2} \tilde{X}^{T} \tilde{X}+\frac{\tilde{P}^{T} \tilde{P}}{2 \gamma}
$$

In the formula, $\quad \tilde{P}=P^{*}-\hat{P}$, from (6) and (9), $\dot{\tilde{X}}$ is derived and substituted into (12): 
$\dot{L}=-\tilde{\mathrm{X}}^{T} K_{\mathrm{p}} \tilde{X}+\tilde{X}^{T} \hat{J} K_{r}^{-1} \mathrm{~F}(\mathrm{t})-\tilde{X}^{T} \hat{J} \hat{K}_{r}^{-1} u_{r}-\frac{\tilde{P}^{T} \dot{\hat{p}}}{\gamma}$

The upper limit of $F(t)$ is $\eta(t)$,therefore, according to (13):

$$
\begin{aligned}
& \dot{L} \leq-\tilde{X}^{T} K_{p} \tilde{X}+\left\|\tilde{X}^{T} \hat{J} \hat{K}_{r}^{-1}\right\| \eta(t)- \\
& \tilde{X}^{T} \hat{J} \hat{K}_{r}^{-1} u_{r}-\frac{\tilde{P}^{T} \dot{\hat{P}}}{\gamma}
\end{aligned}
$$

Suppose $u_{r}$ is described in [10], here $\hat{y}=\hat{\eta} \mu^{T}, \mu=\tilde{X}^{T} \hat{J} \hat{K}_{r}^{-1}$, Substituting $u_{r}, \hat{y}$ and $\mu$ into equation (14) yields:

$$
\begin{aligned}
& \dot{L} \leq-\tilde{X}^{T} K_{p} \tilde{X}+\|\mu\| \eta(t)- \\
& \mu \frac{\hat{y} \hat{\eta}}{\|\hat{y}\|+\lambda e^{-\beta t}}-\frac{\tilde{P}^{T} \dot{\hat{P}}}{\gamma}
\end{aligned}
$$

Equation (15) can be rewritten as follows:

$$
\begin{aligned}
& \dot{L} \leq-\tilde{X}^{T} K_{p} \tilde{X}+\|\hat{y}\|- \\
& \frac{\mu \hat{y} \hat{\eta}}{\|\hat{y}\|+\lambda e^{-\beta t}}+\|\mu\| \tilde{P}^{T} \xi-\frac{\tilde{P}^{T} \dot{\hat{P}}}{\gamma}
\end{aligned}
$$

By (16) available:

$$
\begin{aligned}
& \dot{L} \leq-\tilde{X}^{T} K_{p} \tilde{X}+\frac{\|\hat{y}\| \lambda e^{-\beta t}}{\|\hat{y}\|+\lambda e^{-\beta t}}+\|\mu\| \tilde{P}^{T} \xi \\
& -\frac{\tilde{P}^{T} \dot{\hat{P}}}{\gamma}
\end{aligned}
$$

Consider the adaptive rule $\dot{\hat{P}}=\gamma\|\mu\| \xi$, equation (17) can be simplified as follows:

$$
\dot{L} \leq-\tilde{X}^{T} K_{p} \tilde{X}+\lambda e^{-\beta t}
$$

This indicates that the tracking error $\tilde{X}$ converges to zero asymptotically [14], the vector $\hat{P}$ is bounded. According to the literature [10] can be drawn state vector $Z=\left[\begin{array}{lll}q & \dot{q} & I_{\mathrm{a}}\end{array}\right]^{T}$ is bounded. Therefore, the proposed control law is:

$$
\hat{K}_{b} \hat{r}^{-1} \hat{J}^{-1}(q)\left(\dot{X}_{d}+K_{p} \tilde{X}\right)+u_{r}=v
$$

\section{Simulation Results}

The control law proposed in this paper is simulated by a SCARA robot manipulator. The specific parameters and cost functions are described in [11]. The parameter $\gamma$ is chosen to be 1000, and the initial value of LPs is selected at random. Uncertain boundary $\eta(t)$ can be estimated according to 
(11). $\hat{P}$ on-line adjustment using adaptive rules. In order to make the estimation error as small as possible, We should increase the number of orthogonal functions of vector $\xi$. Fig. 1. shows the tracking performance in the xy plane.

The value of parameter $\gamma$ is chosen to be 1000 , and the initial value of LPs is selected at random. Fig. 2. for the end of the tracking error of the actuator, fulfil requirements.

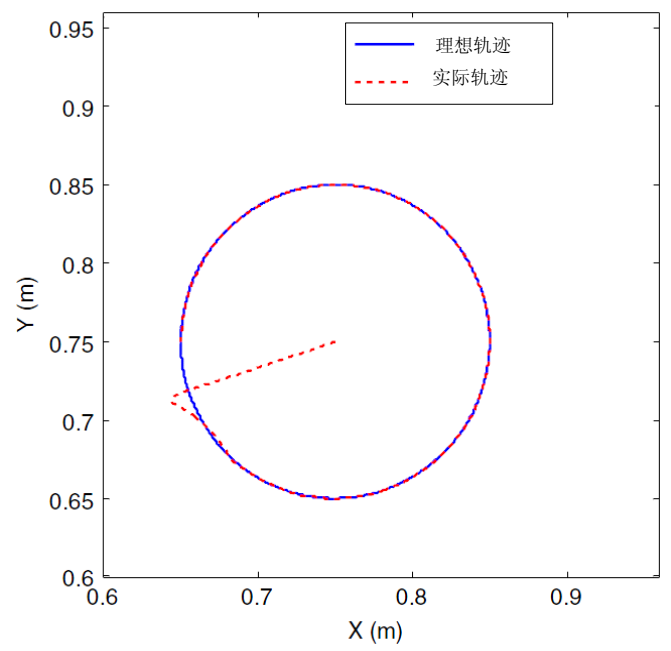

Figure 1. Tracking the performance of the xy plane

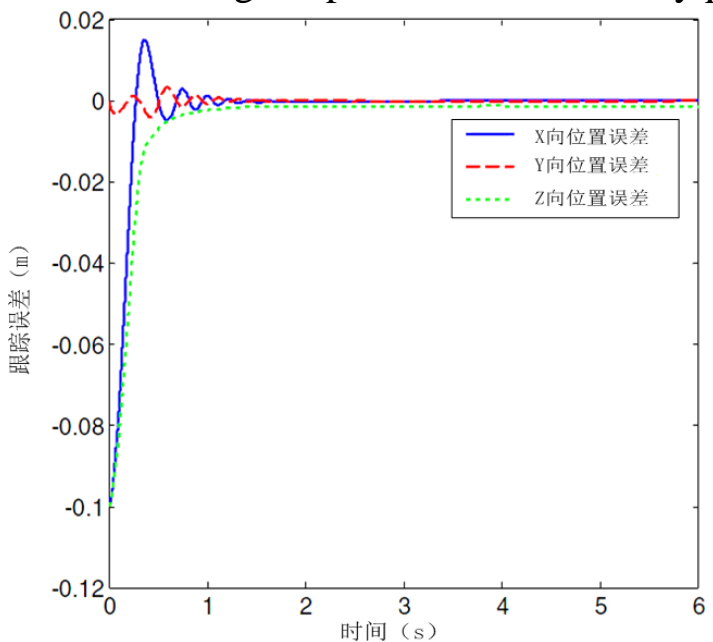

Figure 2. Task space tracking error

As shown in Fig. 3. for the control algorithm proposed control attempts, the motor voltage is smooth and does not exceed the maximum allowable voltage. The change and self-adaptation of Legendre coefficient As shown in Fig.4, Fig. 4. shows that these coefficients are bounded. For the entire controller, the $C_{f}$ value is 0.03709. According to the literature [12], for X, the term 11 is sufficient. Simulation results show that it is necessary to increase the number of LPs. To assess the estimated effect, we increase the size of $\xi$ to $13,15,17$, and 19. For all simulations, $C_{f}$ is approximately 0.03709 . 


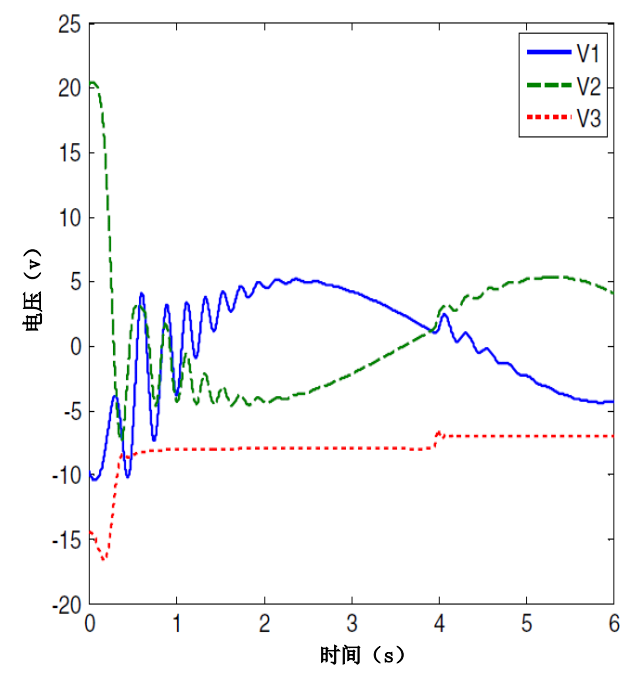

Figure 3. Control of the proposed algorithm

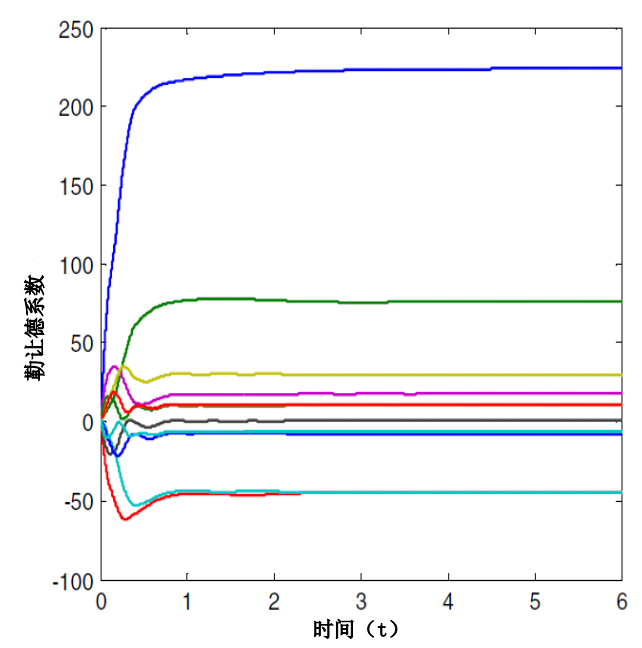

Figure 4. Curve of Legendre coefficient

To evaluate the effect of parametric uncertainties, We have simulated $\hat{J}(q)=0.5 J(q)$ and $\hat{K}_{r}=0.25 K_{r}$. XY plane tracking error and motor voltage as shown in Fig. 5. and Fig. 6., the figure shows that although the parameter uncertainty increases, but the robustness of the controller is to meet the requirements. The value of the cost function $C_{f}$ is increased to 0.06892 .

Effect evaluation of parameter uncertainties: assuming that the robust item $u_{r}$ is 0 , , then the cost function value increases from 0.03709 to 0.06941 . Which shows the effectiveness of the proposed method. 


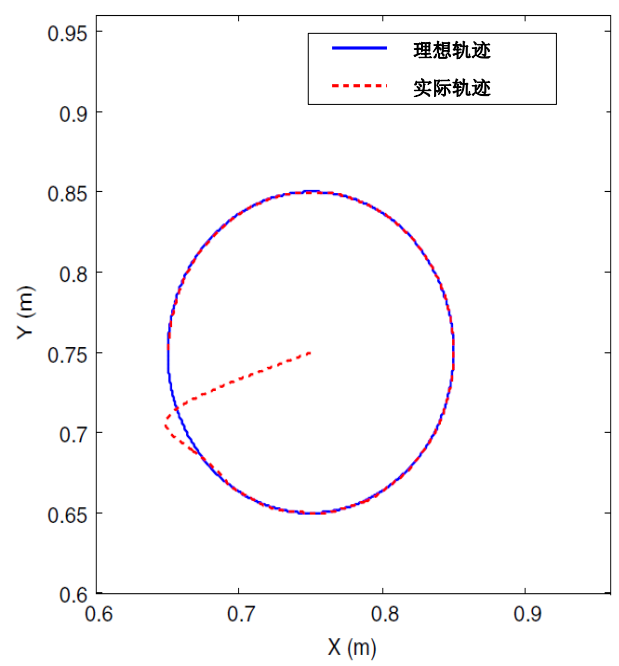

Figure 5. Tracking error in xy plane with increasing parameter uncertainties

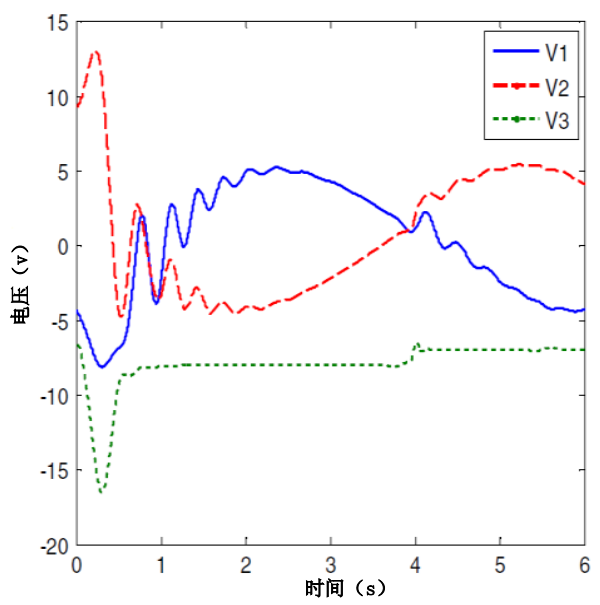

Figure 6. Control attempts in the case of uncertain parameters

\section{Conclusion}

Aiming at the problem that the traditional robust control method needs uncertain boundary as the function of the state system and external disturbance upper limit, and the feedback cost of the controller is expensive and difficult to realize, this paper proposes a control law based on LPs and carries on the simulation analysis. The results show that the advantage of the function approximation technique is that the number of feedbacks is much less than that of the traditional robust control method.

\section{Acknowledgements}

This work was financially supported by Jilin Scientific and Technological Development Program (20140101185JC, 20140101206JC-16), Jilin City Scientific and Technological Development Program (201464048), Scientific and Technological Research Program of Jilin Educational Committee during the "12th Five-Year Plan" (2015-149, 2015-137),Exploring Practice of C Language Program Design" Case Teaching for Professional Application(XJQN2016035), Research and Practice of Self-exploration Learning Model under the Background of Integration and Innovation - Research on VB Program Design Based on Non-Computer 
Specialty(XJYB2016025),A Study on Evolving Mechanism and Governance Strategy of Public Safety Public Opinion Ecosystem(2016JD44).

\section{References}

[1] LIU Fu-Cai, GAO Jing-Fang, JIA Xiao-Jing.Neural Network Control of Task Space for Flexible Joint Space Manipulators Considering the Influence of Gravity [J]. Journal of Astronautics 2015; 36 (12): 1391-1397.

[2] Chen Zhiyong, Chen Li. Adaptive Backstepping Control of Flexible Joint Space Robot Based on Neural Network [J]. Engineering Mechanics 2013; 30 (4): 397-401.

[3] Abdallah, C., Dawson, D., Dorato, P., Jamshidi, M .: Survey of robust control for rigid robots. IEEE Control Syst. Mag. 11, 24-30 (1991)

[4] Corless, M.J .: Control of uncertain nonlinear systems. ASME Trans. J. Dyn. Syst. Meas. Control 115 (2B), 362-372 (1993)

[5] Fateh, M.M .: On the voltage-based control of robot manipulators. Int. J. Control Autom. Syst. 6 (5), 702-712 (2008)

[6] Modeling and voltage control of three-degree-of-freedom permanent magnet spherical motor [J]; JOURNAL OF JILIN UNIVERSITY (ENGINEERING EDITION) 2014; 44 (2), 560-566 (in Chinese with English abstract)

[7] WANG Peng-Jun, ZHANG Xue-Long, ZHANG Yue-Jun.Design of High Robust PUF Circuit Based on Optimal Control Voltage [J] .Acta Electronica Sinica 2015; 43 (5) 907-910

[8] HAN Pu, YU Ping, WANG Dongfeng, HUANG Baohai.Adaptive Inverse Control Method Based on Support Vector Regression [J]. Journal of North China Electric Power University 2006; 33 (3): 31-35.

[9] Kreyszig, E.: Advanced Engineering Mathematics. Wiley,NY (2007)

[10] Talole, S.E., Kolhe, J.P., Phadke, S.B.: Extended-stateobserver-based control of flexible-joint system with experimental validation. IEEE Trans. Ind. Electron. 57(4), 1411-1419 (2010)

[11] Chen, W.H., Ballance, D.J., Gawthrop, P.J., O'Reilly, J.: A nonlinear disturbance observer for robotic manipulators. IEEE Trans. Ind. Electron. 47(4), 932-938 (2000)

[12]Chien, M.C., Huang, A.C.: Adaptive impedance controller design for flexible-joint electrically-driven robots without computation of the regressor matrix. Robotica 30, 133-144 (2012) 